rescue team need to be improved. The discipline system of disaster medicine in China is still lagging behind, and it is still unable to meet the needs of the rescue situation at home and abroad.

Discussion: Disaster medicine is the area of medical specialization serving the dual areas of providing health care to disaster survivors and providing medically related disaster preparation, disaster planning, disaster response, and disaster recovery leadership throughout the disaster life cycle. It requires multidisciplinary intervention, integration, and application. A forwardlooking perspective must be strengthened on the discipline and team building of disaster medicine.

Prehosp. Disaster Med. 2019;34(Suppl. 1):s21-s22

doi:10.1017/S1049023X19000621

\section{Reviewing Disasters: Hospital Evacuations in the United States from 2000 to 2017}

Miss. Aishwarya Sharma ${ }^{1}$, Dr. Sharon Mace ${ }^{2}$

1. Ohio University Heritage College of Osteopathic Medicine, Warrensville Heights, United States

2. Cleveland Clinic Lerner College of Medicine at Case Western Reserve University, Cleveland, United States

Introduction: Between 2000 to 2017, there were over 150 hospital evacuations in the United States. Data received from approximately 35 states were primarily concentrated in California, Florida, and Texas. This analysis will provide disaster planners and administrators statistics on hazards that cause disruptions to hospital facilities.

Aim: The aim of this study is to investigate US hospital evacuations by compiling the data into external, internal, and manmade disasters thus creating a risk assessment for disaster planning. Methods: Hospital reports were retrieved from LexisNexis, Google, and PubMed databases and categorized according to evacuees, duration, location, and type. These incidents were grouped into three classifications: external, internal, and manmade. Both partial and full evacuations were included in the study design.

Results: There were a total of 154 reported evacuations in the United States. 110 (71\%) were due to external threats, followed by $24(16 \%)$ man-made threats, and $20(13 \%)$ internal threats. Assessing the external causes, 60 (55\%) were attributed to hurricanes, $21(19 \%)$ to wildfires, and $8(7 \%)$ to storms. From the internal threats, $8(40 \%)$ were attributed to hospital fires and 4 (20\%) chemical fumes. From the man-made threats, $6(40 \%)$ were attributed to bomb threats and $4(27 \%)$ gunmen. From the 20 total reported durations of evacuations, 9 (45\%) lasted between 2 to 11:59 hours, 6 (30\%) lasted over 24 hours, and $5(25 \%)$ lasted up to $1: 59$ hours.

Discussion: Over 70\% of hospital evacuations in the US were due to natural disasters. Compared to 1971-1999, there was an increase in internal and man-made threats. Exact statistics on evacuees, durations, injuries, and mortality rates were unascertainable due to a lack of reporting. It is critical to implement a national registry to report specifics on incidences of evacuations to further assist with disaster and infrastructure planning.

Prehosp. Disaster Med. 2019;34(Suppl. 1):s22

doi:10.1017/S1049023X19000633

\section{"We Just Want to Help" - Nonprofits Contributions to Community Resilience in the Disaster Space \\ Ms. Fiona Roberts, Emeritus Professor Frank Archer, \\ Dr. Caroline Spencer \\ MUARC, Monash University, Clayton, Australia}

Introduction: The National Strategy for Disaster Resilience (NSDR) characterizes resilient communities as having strong disaster and financial mitigation strategies, strong social capacity, networks, and self-reliance. Nonprofit organizations (NPOs) embrace many characteristics of a disaster resilient community. NPOs do not operate for the profit of individual members. Community groups like Lions and Rotary Club have long histories, and while not established to respond to disasters, they frequently have heavy involvement in preparing for or recovering from, disasters.

Aim: The study aims to address the question, "What is the potential role of nonprofit organizations in building community resilience to disasters?"

Methods: An applied research project was carried out, using theories of resilience, social capital, and the Sendai framework to conceptualize the frameworks and guide the process. Qualitative research methods, thematic analysis, and case studies helped identify Lions, Rotary, and Neighbourhood Houses Victoria strengths, barriers, and enablers.

Results: Research demonstrated how NPOs made significant contributions to building communities' resilience to disasters. NPOs facilitate three Sendai guiding principles of engaging, empowering, and enabling the community to build disaster resilience. Actions included raising awareness to disaster risk, reducing disaster risk, helping prepare for disasters, and contributing to long term disaster recovery. NPO strengths included local knowledge, community trust, and connections, which matched characteristics listed in the NSDR for a disaster resilient community. However, barriers to participation included traditional emergency services ignoring NPOs, lack of role definition, and lack of perceived legitimacy.

Discussion: As the first Australia research to scientifically analyze the contributions of these NPOs to build community resilience, before, during and after disaster, this study enhances understanding and recognition of NPOs and assists in identifying means to facilitate their disaster resilience activities and place them more effectively within Emergency Management strategic processes. Greater utilization of such assets could lead to better community outcomes.

Prehosp. Disaster Med. 2019;34(Suppl. 1):s22

doi:10.1017/S1049023X19000645 